\title{
Nomograms Predicting Platinum Sensitivity, Progression-Free Survival, and Overall Survival Using Pretreatment Complete Blood Cell Counts in Epithelial Ovarian Cancer
}

\section{E Sun Paik, MD \\ Insuk Sohn, PhD² \\ Sun-Young Baek, MSc \\ Minhee Shim, MD' \\ Hyun Jin Choi, MD \\ Tae-Joong Kim, MD, PhD ${ }^{1}$ \\ Chel Hun Choi, MD, PhD' \\ Jeong-Won Lee, MD, $P h D^{1}$ \\ Byoung-Gie Kim, MD, PhD \\ Yoo-Young Lee, MD, $\mathrm{PhD}^{3}$ \\ Duk-Soo Bae, MD, PhD'}

\author{
${ }^{1}$ Department of Obstetrics and Gynecology, \\ Samsung Medical Center, Sungkyunkwan \\ University School of Medicine, Seoul, \\ ${ }^{2}$ Biostatistics and Clinical Epidemiology \\ Center, Samsung Medical Center, \\ Seoul, Korea, ${ }^{3}$ Division of Gynecologic \\ Oncology, Princess Margaret Cancer Centre, \\ University Health Network, Toronto, \\ ON, Canada
}

\begin{abstract}
Purpose
This study was conducted to evaluate the prognostic significance of pre-treatment complete blood cell count (CBC), including white blood cell (WBC) differential, in epithelial ovarian cancer (EOC) patients with primary debulking surgery (PDS) and to develop nomograms for platinum sensitivity, progression-free survival (PFS), and overall survival (OS).
\end{abstract}

\section{Materials and Methods}

We retrospectively reviewed the records of 757 patients with EOC whose primary treatment consisted of surgical debulking and chemotherapy at Samsung Medical Center from 2002 to 2012. We subsequently created nomograms for platinum sensitivity, 3-year PFS, and 5-year OS as prediction models for prognostic variables including age, stage, grade, cancer antigen 125 level, residual disease after PDS, and pre-treatment WBC differential counts. The models were then validated by 10 -fold cross-validation (CV).

\section{Results}

In addition to stage and residual disease after PDS, which are known predictors, lymphocyte and monocyte count were found to be significant prognostic factors for platinum-sensitivity, platelet count for PFS, and neutrophil count for OS on multivariate analysis. The area under the curves of platinum sensitivity, 3-year PFS, and 5-year OS calculated by the 10-fold CV procedure were $0.7405,0.8159$, and 0.815 , respectively.

\section{Conclusion}

Prognostic factors including pre-treatment CBC were used to develop nomograms for platinum sensitivity, 3-year PFS, and 5-year OS of patients with EOC. These nomograms can be used to better estimate individual outcomes.

\section{Key words}

Nomograms, Prognosis, Ovarian neoplasms
Correspondence: Duk-Soo Bae, MD, PhD Department of Obstetrics and Gynecology, Samsung Medical Center, Sungkyunkwan University School of Medicine, 81 Irwon-ro, Gangnam-gu, Seoul 06351, Korea

Tel: 82-2-3410-3511

Fax: 82-2-3410-0630

E-mail: ds123.bae@samsung.com

Co-correspondence: Yoo-Young Lee, MD, PhD Division of Gynecologic Oncology,

Princess Margaret Cancer Centre, University

Health Network, 610 University Ave.,

Toronto, ON, Canada

Tel: 1-647-642-0787

Fax: 1-416-946-6563

E-mail:heyu0a@gmail.com

Received July 3, 2016

Accepted September 16, 2016

Published Online September 27, 2016 


\section{Introduction}

Epithelial ovarian cancer (EOC) is one of the leading causes of death in females with gynecological malignancies [1]. Most patients respond to primary treatment, and $75 \%$ of patients reach complete response. However, $40 \%-60 \%$ of all patients with EOC and 75\% of those with advanced stage disease will eventually experience recurrence $[2,3]$. Accurate estimation of survival for patients with EOC is important because prognosis is a determinate of treatment aggressiveness tailored to the individual situation. Patients who experience recurrence after 6 months from the end of primary chemotherapy are classified as platinum sensitive, and currently, platinum sensitivity is considered an important factor for predicting survival outcomes [4]. Predicting platinum sensitivity in patients with EOC may play an important role in establishing treatment plans.

Previous studies demonstrated several biological markers as significant prognostic factors for oncologic outcomes after treatment. Laboratory systemic inflammatory response markers have been studied as prognostic factors in a variety of cancers [5-7]. Paraneoplastic lymphocytopenia, leukocytosis, and thrombocytosis are significant prognostic factors in many solid tumors. However, the level of contribution of each biological marker to oncological outcomes such as platinum sensitivity and survival in EOC is not fully understood.

This study was conducted to evaluate the clinical impact of pre-treatment complete blood cell count $(\mathrm{CBC})$ including white blood cell (WBC) differential components as prognostic factors for platinum-sensitivity on EOC patients with primary debulking surgery (PDS) and to develop nomograms for platinum sensitivity, 3-year progression-free survival (PFS), and 5-year overall survival (OS) with prognostic CBC components and known prognostic clinical parameters.

\section{Materials and Methods}

\section{Patients}

After obtaining Institutional Review Board approval (IRB file No. 2015-06-092), data were collected from Samsung Medical Center for patients with EOC who were treated from January 2002 to December 2012. We identified 757 patients whose primary treatment consisted of PDS and adjuvant chemotherapy. Patients who underwent neo-adjuvant chemotherapy and interval debulking surgery, those who had a transfusion within 2 weeks prior to PDS, and patients with concurrent cancer other than ovarian cancer were excluded from the study. Patients who received intraperitoneal or dose-dense chemotherapy were not included in this study.

\section{Treatment and follow-up}

Standard primary surgical treatment consisted of hysterectomy, bilateral salpingo-oophorectomy, omentectomy, retroperitoneal (pelvic and para-aortic) lymphadenectomy, and any tumorectomy of metastatic lesions, if applicable. Peritoneal washing was routinely conducted. If any abnormalities were identified, peritoneal biopsies from different sites were performed. Early stage EOC patients who wanted fertility saving received fertility saving surgery with/without chemotherapy as primary treatment.

Within 2 weeks prior to PDS, patients were routinely required to undergo basic preoperative evaluation including complete blood count. After PDS, patients started the first cycle of platinum-based combination chemotherapy, which was repeated every 3 weeks for six cycles. Abdominopelvic computed tomography (CT) scan was routinely performed after first three cycles of chemotherapy and after six cycles of first-line treatment. Following primary treatment, patients were assessed by physical examination, $\mathrm{CBC}$, and chemistry with serum tumor markers, including cancer antigen 125 (CA-125) measurements, every 3 months for the first 2 years and twice per year thereafter. Chest radiography and abdominopelvic CT scan (or alternatively abdominopelvic magnetic resonance imaging) were performed every 6 months for the first 3 years and every 12 months thereafter. Additional diagnostic procedures were performed according to specific clinical suspicions. If recurrence was suspected with symptoms or CA-125 elevation, additional imaging studies were performed. Recurrence may have been detected by imaging studies with or without CA-125 elevation. Response to chemotherapy was assessed and recorded according to the Response Evaluation Criteria in Solid Tumors (RECIST).

OS was defined as the time from diagnosis to the date of the patient's death or loss to follow-up. PFS was defined as the time from diagnosis to the date of recurrence or loss to follow-up. Disease-free interval (DFI) was defined as the time from the end of primary treatment to the date of recurrence.

\section{Statistical analysis}

A multivariate logistic regression model with stepwise variable selection using Akaike's information criterion (AIC) was employed to identify factors predictive of platinum sensitivity. A multivariate Cox regression model with stepwise variable selection using AIC was used to identify prognostic factors for OS and PFS. The proportional hazards assumption was assessed using the method proposed by Grambsch and 
Therneau [8] and the linearity assumption was checked using a penalized smoothing spline method. Two assumptions for neutrophil, lymphocyte, monocyte, platelet, and age were satisfied (S1 and S2 Tables). Prognostic factors identified by multivariate analysis were used to create a nomogram to predict platinum sensitivity, 3-year PFS, and 5-year OS. We validated each nomogram using 10 -fold cross-validation (CV) [9]. We then constructed a receiver operating characteristic (ROC) curve for platinum-sensitivity and calculated the area under the curve (AUC). The optimal cut-off point for predicted probability was determined by maximizing the Youden index [10]. Time-dependent ROC curves were constructed using the Nearest Neighbor Estimation method for 3-year PFS and 5-year OS [11] and AUCs were calculated. Statistical analyses were performed using R 3.0.3 (Vienna, Austria; http://www.R-project.org).

Prognostic variables including age, histology, stage, tumor grade, residual disease after PDS, and preoperative CBC (hemoglobin, WBC differential [neutrophil count, lymphocyte count, monocyte count], and platelet count) were used in the analysis. Platinum sensitivity was included in analysis of the 5-year OS. Patients with non-serous carcinoma were combined and compared to those with serous carcinoma. Residual disease after PDS was categorized based on size (no residual, $\leq 1 \mathrm{~cm}$, and $>1 \mathrm{~cm}$ ). Platinum sensitivity was classified as platinum-resistant or platinum-sensitive (DFI $\leq$ 6 months, DFI $>6$ months), or unknown for platinum sensitivity. Patients with insufficient observation time to determine platinum sensitivity, as well as those who did not receive platinum-based chemotherapy as primary treatment were classified as unknown for platinum sensitivity.

\section{Results}

Data from the records of a total of 757 patients with EOC who were treated at Samsung Medical Center were analyzed. Patient demographic and clinical characteristics are listed in Table 1. There were 430 cases of cancer recurrence and 276 cases of cancer-specific death with a median follow-up of 51 months (range, 4 to 156 months). The majority of patients had stage III $(56.5 \%)$, grade 3 disease $(70.9 \%)$ of serous type $(63.8 \%)$. There was no residual disease in 277 patients $(36.6 \%)$ in the whole cohort, nor in $22.2 \%(115 / 517)$ of those in advanced stage. Of all patients, 647 patients $(85.5 \%)$ were platinum-sensitive.

Multivariate logistic regression analysis for platinum sensitivity and multivariate Cox modeling for PFS and OS were used to evaluate independent prognostic factors and estimate their effects for all patients. Stage and residual disease
Table 1. Patient demographic and clinical characteristics

\begin{tabular}{|c|c|}
\hline Characteristic & No. $(\%)(n=757)$ \\
\hline Age, median (range, yr) & $52(15-84)$ \\
\hline CA-125, median (range, U/mL) & $522.75(1.0-100,080.0)$ \\
\hline \multicolumn{2}{|l|}{ Stage $(\%)$} \\
\hline I & $162(21.4)$ \\
\hline II & $78(10.3)$ \\
\hline III & $428(56.5)$ \\
\hline IV & $89(11.8)$ \\
\hline \multicolumn{2}{|l|}{ Grade $(\%)$} \\
\hline 1 & $61(8.1)$ \\
\hline 2 & $159(21.0)$ \\
\hline 3 & $537(70.9)$ \\
\hline \multicolumn{2}{|l|}{ Histology (\%) } \\
\hline Serous & $483(63.8)$ \\
\hline Endometrioid & $80(10.6)$ \\
\hline Mucinous & $62(8.2)$ \\
\hline Clear cell & $59(7.8)$ \\
\hline Transitional & $28(3.7)$ \\
\hline Mixed & $31(4.1)$ \\
\hline Others & $14(1.8)$ \\
\hline \multicolumn{2}{|l|}{ Residual disease after PDS (\%) } \\
\hline No residual & $277(36.6)$ \\
\hline$\leq 1 \mathrm{~cm}$ & $284(37.5)$ \\
\hline$>1 \mathrm{~cm}$ & $196(25.9)$ \\
\hline \multicolumn{2}{|l|}{ Platinum-sensitivity $(\%)$} \\
\hline Platinum resistant & $110(14.5)$ \\
\hline Platinum sensitive & $616(81.4)$ \\
\hline Unknown & $31(4.1)$ \\
\hline \multicolumn{2}{|c|}{$\begin{array}{l}\text { Pretreatment complete blood count, } \\
\text { median (range) }\end{array}$} \\
\hline Hemoglobin (g/dL) & $12.2(7.6-15.6)$ \\
\hline Platelet count $\left(\times 10^{3} / \mu \mathrm{L}\right)$ & $304(63-764)$ \\
\hline Neutrophil count $\left(\times 10^{3} / \mu \mathrm{L}\right)$ & $4.355(0.730-27.250)$ \\
\hline Lymphocyte count $\left(\times 10^{3} / \mu \mathrm{L}\right)$ & $1.584(0.401-3.883)$ \\
\hline Monocyte count $\left(\times 10^{3} / \mu \mathrm{L}\right)$ & $0.427(0.089-1.756)$ \\
\hline
\end{tabular}

CA-125, cancer antigen 125; PDS, primary debulking surgery.

after PDS were significant predictors of OS, PFS, and platinum sensitivity upon multivariate analysis. In addition, some of the preoperative $\mathrm{CBC}$ components were found to be significant prognostic factors.

Analyses for platinum sensitivity are shown in S3 Table. Among the significant variables identified upon univariate analysis, preoperative lymphocyte count and monocyte count, stage, histology, and residual disease after PDS were significant predictors of platinum sensitivity. Platelet count, stage, grade, and residual disease after PDS appeared to be significant predictors of 3-year PFS upon multivariate analy- 


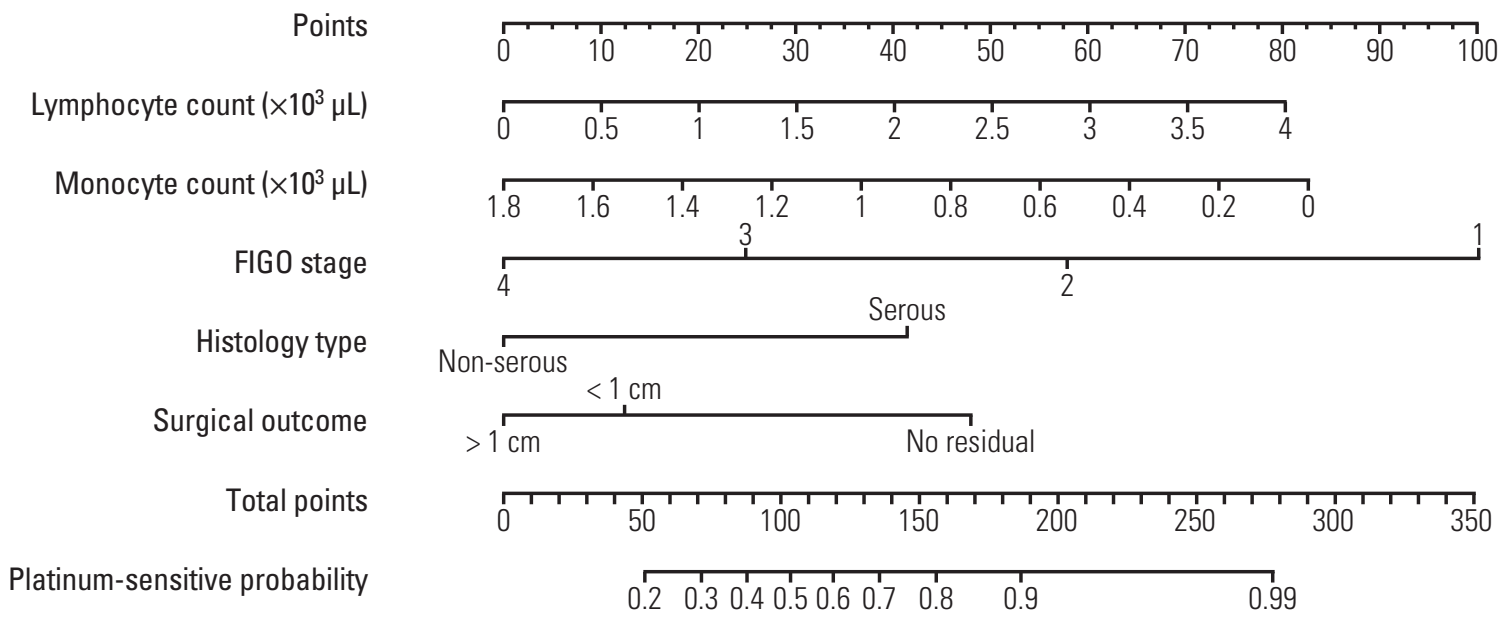

Fig. 1. Nomogram for predicting platinum sensitivity. FIGO, International Federation of Gynecology and Obstetrics.

Points

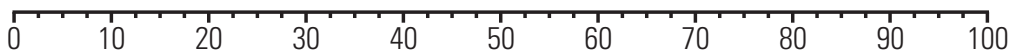

Platelet count $\left(\times 10^{3} \mu \mathrm{L}\right)$

FIGO stage

$\begin{array}{lllllllll}0 & 100 & 200 & 300 & 400 & 500 & 600 & 700 & 800\end{array}$

Grade

Surgical outcome

Total points

Linear predictor

3-Year progression-free

survival probability
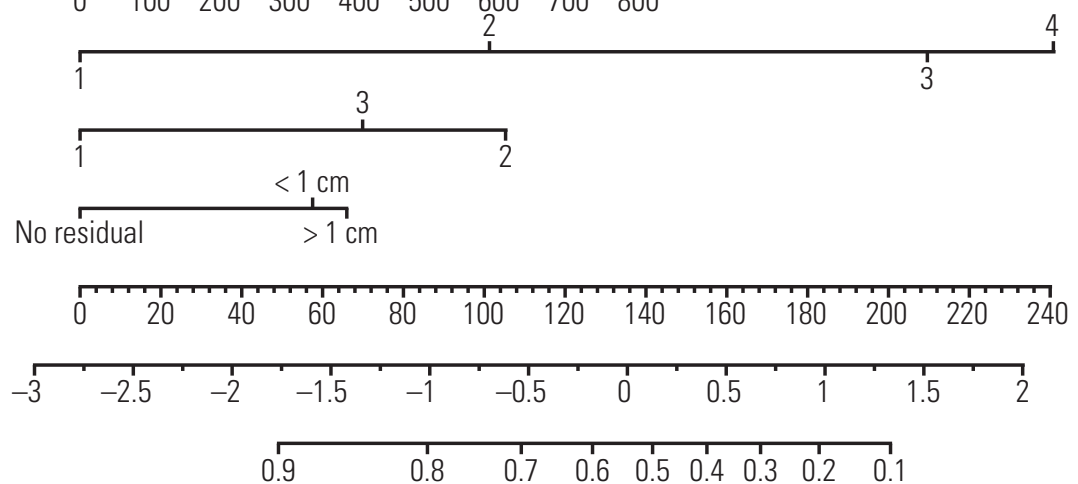

Fig. 2. Nomogram for predicting 3-year progression-free survival. FIGO, International Federation of Gynecology and Obstetrics.

sis (S4 Table). Pretreatment neutrophil count, stage, residual disease after PDS, and platinum sensitivity were significant prognostic factors for 5-year OS (S5 Table).

Nomograms were developed to account for the importance of each clinical prognostic variable. Nomograms were created using the multivariate logistic regression model for platinum sensitivity and multivariate Cox regression models for 3-year PFS and 5-year OS (Figs. 1-3). Use of the nomograms is described in the figure captions. For example, in the nomogram for platinum sensitivity, the user should find the patient's stage on the Stage axis, then draw a straight line upward to the Points axis to determine how many points toward progression the patient receives for stage. This should be done again for the other axes, with the user drawing a straight line upward toward the Points axis each time. The points received for each predictor are then summed and the sum is found on the total points axis. The user should then draw a straight line down to the platinum sensitivity probability axis to find the patient's probability of platinum sensitivity.

The AUCs of platinum sensitivity, 3-year PFS, and 5-year OS calculated using the 10 -fold CV procedures were 0.7405 , 
Points

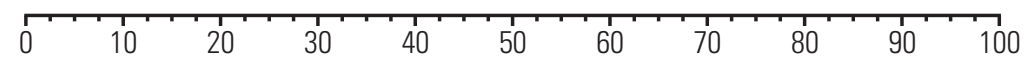

Neutrophil count $\left(\times 10^{3} \mu \mathrm{L}\right)$

\begin{tabular}{lllllllllllllll}
\hline 0 & 2 & 4 & 6 & 8 & 10 & 12 & 14 & 16 & 18 & 20 & 22 & 24 & 26 & 28
\end{tabular}

Age

FIGO stage
Surgical outcome
Platinum sensitivity

FIGO stage
Surgical outcome
Platinum sensitivity

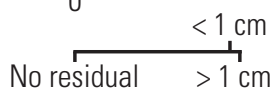

030456075

Total points

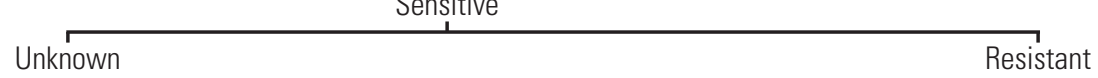

Linear predictor

$\begin{array}{llllllllllllll} & 0 & 20 & 40 & 60 & 80 & 100 & 120 & 140 & 160 & 180 & 200 & 220 & 240\end{array}$

$\begin{array}{lllllllllllllll}-2.5 & -2 & -1.5 & -1 & -0.5 & 0 & 0.5 & 1 & 1.5 & 2 & 2.5 & 3 & 3.5 & 4\end{array}$

5-Year overall survival probability

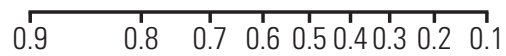

Fig. 3. Nomogram for predicting 5-year overall survival. FIGO, International Federation of Gynecology and Obstetrics.

0.8159 , and 0.815 , respectively. The optimal cut-off value of predicted probability for platinum sensitivity was 0.8594 , and the sensitivity and specificity were 0.6318 and 0.8073 , respectively. Calibration curves for the platinum sensitivity, 3-year PFS and 5-year OS nomograms are shown in Fig. 4. The nomograms for 3-year PFS and 5-year OS were well calibrated.

\section{Discussion}

In this study, we evaluated pre-treatment $\mathrm{CBC}$ as a prognostic factor for EOC treated with PDS and adjuvant chemotherapy and its impact on prognosis. We found that platelet count, neutrophil count, monocyte count, and lymphocyte count were significantly associated with platinum sensitivity, PFS, and OS. Other common prognostic factors identified in our study included stage and residual disease after PDS. Therefore, we sought to develop nomograms that would include these prognostic factors for platinum sensitivity, PFS, and OS. The nomograms were validated for platinum sensitivity, 3-year PFS, and 5-year OS.

There are number of risk factors associated with survival in EOC. Most of the previous risk analyses only provide information regarding individual risk factors based on univariate and multivariate analyses. Traditional risk stratification strategies assign all risk factors the same weight, which may cause bias. In contrast, a nomogram provides parametric information for end-point prediction by integrating mul- tiple weighted risk factors. After summing all of the points for each factor, results can be translated into information regarding survival. This information can facilitate discussion between the physician and the patient and guide clinical care. Nomograms have been constructed to predict various clinical end points for patients with different types of cancer [12-14]. A nomogram should theoretically be more specific to each individual patient and thus able to predict specific clinical endpoints more accurately.

Paraneoplastic lymphocy topenia, leukocytosis, and thrombocytosis are well-known prognostic factors for many solid tumors. Various studies explaining the prognostic significance of inflammatory markers from peripheral blood including components from pretreatment $\mathrm{CBC}$ s have been reported [15-17]. Cancer cells secrete cytokines such as interleukin 6, which directly and indirectly stimulate platelet production in tumor cells, thereby enhancing proliferation and metastasis [17]. Tumors are known to induce neutrophilic differentiation and stimulate angiogenesis and cell proliferation by producing chemokines, cytokines, and prostaglandins [18]. Previous reports demonstrated that a higher neutrophil count and lower lymphocyte count predict poorer survival in EOC $[7,16]$. Lymphocytes showed greater decreases in patients with higher stage, ascites, and residual cancer after PDS in EOC [19]. Moreover, significantly lower lymphocyte counts and higher neutrophil counts were associated with greater tumor grade, advanced stage, and presence of ascites [20]. These associations between WBC differential counts and tumor aggressiveness may partially explain the correlation with survival outcomes. A recent study demonstrated that elevated peripheral blood mono- 
A
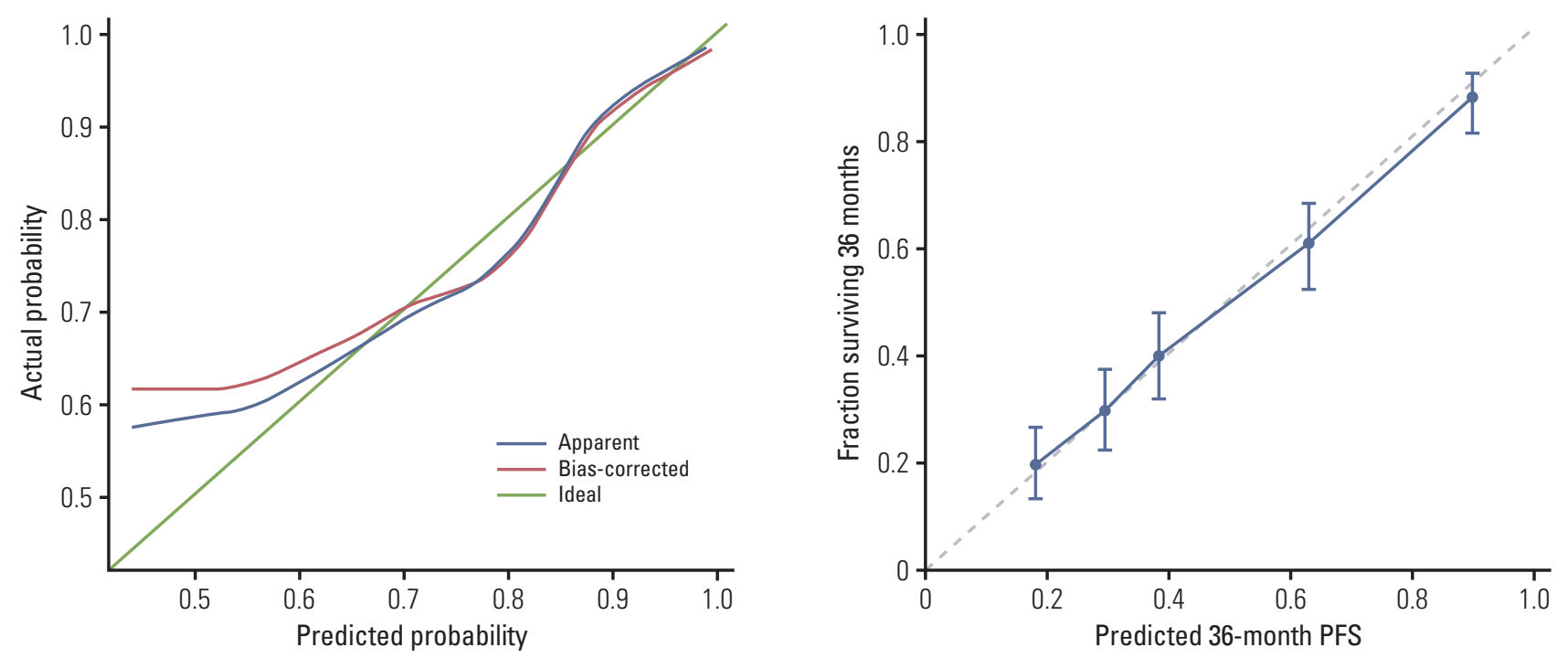

C

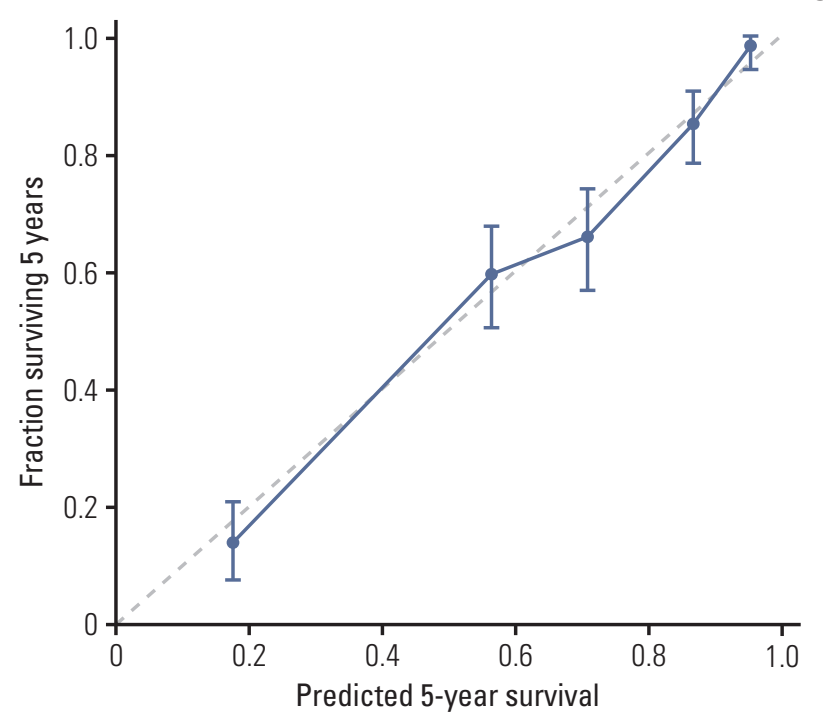

Fig. 4. Calibration plot for platinum sensitivity (A), progression-free survival (PFS) nomogram model (B), and overall survival (C) nomogram model.

cyte count was associated with worse OS, and that monocytes are associated with increased adrenergic signaling via monocyte chemotactic protein 1 , which facilitates tumor progression in EOC [21,22]. Despite evidence that factors from WBC differential counts are predictors of prognosis for different cancers including EOC, larger studies with greater detail on patient profiles, tumor features, and treatment will be necessary to demonstrate that these factors are truly independent predictors.
To the best of our knowledge, this is the first study presenting prognostic nomograms for the endpoint of platinum sensitivity with pre-treatment $\mathrm{CBC}$ including WBC differential counts. Previous reports of survival models in EOC have been based on variables such as stage, residual disease, grade, histology, age, and performance status [23-25]. Furthermore, a number of studies demonstrated laboratory markers from routine testing, such as $\mathrm{CBC}$, as prognostic variables $[13,26-28]$. Our study demonstrated that, in addi- 
tion to the previously published prognostic variables for EOC, WBC differential counts (lymphocyte, monocyte, and neutrophil counts) of pre-treatment CBC may be considered prognostic factors for platinum sensitivity and survival in EOC. We expected minimal bias associated with using pretreatment $\mathrm{CBC}$ since it is commonly performed in a majority of institutions and gives objective values. Based on patient clinicopathologic information and components of pretreatment $\mathrm{CBC}$, the nomogram could be used to estimate platinum sensitivity, 3-year PFS, and 5-year OS rates. With nomograms applied to clinical management, clinicians will be able to apply different strategies for subsequent systemic anti-tumor therapy and follow-up interval based on the platinum sensitivity in EOC patients with PDS.

It should be noted that our study had several limitations. Specifically, its retrospective, single-center nature may have resulted in unmeasured confounding factors. When analyzing variables, not all known prognostic factors identified in other prognostic models, such as the volume of ascites and performance status $[13,23,25,29]$, were included because of a substantial lack of data in the medical records. We also did not have sufficient data describing specific circumstances for each patient included in the unknown platinum sensitivity category to explain the reason for the lower hazard ratio. Moreover, the fact that systemic conditions other than cancer (e.g., inflammatory disease or infection status) may have affected the pretreatment $\mathrm{CBC}$ status should not be overlooked. Finally, preoperative comorbidities were not assessed in this analysis.

Although previous studies have shown that factors from WBC differential counts are predictors of prognosis for EOC, the mechanism of results cannot be clearly explained. In addition, our finding that grade reversely affected the survival outcome is difficult to explain. In multivariate analysis for 3-year PFS, grade 3 showed a lower hazard ratio than grade 2 , contrary to univariate analysis. In a previous study, no difference was found in clinical outcomes between grade 2 and 3 of serous ovarian cancer, and in serous ovarian cancer, a 2-tier grade system (high grade vs. low grade) was reportedly more accurate for predicting clinical outcomes than a International Federation of Gynecology and Obstetrics (FIGO) 3-tier (grade 1, 2, and 3) system [30]. In our study, we used a FIGO 3-tier system because we included not only the results of serous type, but also other histologic types. It is possible that the lack of a survival difference between grade 2 and 3 serous types might have affected the results of the hazard ratio for grade upon multivariate analysis.

In our study, information regarding dose, schedule, and toxicities of the adjuvant chemotherapy or surgical complications, which may be associated with survival, is lacking because of the deficit of associated information owing to this study's retrospective nature. A larger number of patient pro- files and detailed information will be needed for definite results in the future. Although internally validated, the nomogram needs to be externally validated before it can be generally accepted for clinical application.

\section{Conclusion}

In summary, our multivariate analysis identified WBC differential counts on pre-treatment CBC (neutrophil, monocyte, and lymphocyte counts) as prognostic factors in addition to stage and residual disease after PDS in patients with EOC that was primarily treated with PDS and adjuvant chemotherapy. These prognostic factors allowed development of nomograms predicting platinum sensitivity, 3-year PFS, and 5-year OS. Such nomograms could be used to better estimate outcomes for individual patients.

\section{Electronic Supplementary Material}

Supplementary materials are available at Cancer Research and Treatment website (http://www.e-crt.org).

\section{Conflicts of Interest}

Conflict of interest relevant to this article was not reported.

\section{Acknowledgments}

This study was supported by grants from the National R\&D Program for Cancer Control, Ministry for Health, Welfare and Family Affairs, Republic of Korea (1520100), Korean Health Technology R\&D Project, Ministry for Health \& Welfare, Republic of Korea (HI14C1940), Basic Science Research Program through the National Research Foundation of Korea (NRF) funded by the Ministry of Education (2013R1A1A 2013612), and the Korea Health Technology R\&D Project through the Korea Health Industry Development Institute (KHIDI) funded by the Ministry of Health \& Welfare, Republic of Korea (HI14C3418). 


\section{References}

1. Siegel RL, Miller KD, Jemal A. Cancer statistics, 2015. CA Cancer J Clin. 2015;65:5-29.

2. Vaidya AP, Curtin JP. The follow-up of ovarian cancer. Semin Oncol. 2003;30:401-12.

3. Cotte E, Glehen O, Mohamed F, Lamy F, Falandry C, Golfier $\mathrm{F}$, et al. Cytoreductive surgery and intraperitoneal chemohyperthermia for chemo-resistant and recurrent advanced epithelial ovarian cancer: prospective study of 81 patients. World J Surg. 2007;31:1813-20.

4. Markman M, Rothman R, Hakes T, Reichman B, Hoskins W, Rubin S, et al. Second-line platinum therapy in patients with ovarian cancer previously treated with cisplatin. J Clin Oncol. 1991;9:389-93.

5. Hirashima K, Watanabe M, Shigaki H, Imamura Y, Ida S, Iwatsuki M, et al. Prognostic significance of the modified Glasgow prognostic score in elderly patients with gastric cancer. J Gastroenterol. 2014;49:1040-6.

6. Oh BS, Jang JW, Kwon JH, You CR, Chung KW, Kay CS, et al. Prognostic value of $\mathrm{C}$-reactive protein and neutrophil-to-lymphocyte ratio in patients with hepatocellular carcinoma. BMC Cancer. 2013;13:78.

7. Bishara S, Griffin M, Cargill A, Bali A, Gore ME, Kaye SB, et al. Pre-treatment white blood cell subtypes as prognostic indicators in ovarian cancer. Eur J Obstet Gynecol Reprod Biol. 2008;138:71-5.

8. Grant S, Chen YQ, May S. Performance of goodness-of-fit tests for the Cox proportional hazards model with time-varying covariates. Lifetime Data Anal. 2014;20:355-68.

9. Simon RM, Subramanian J, Li MC, Menezes S. Using crossvalidation to evaluate predictive accuracy of survival risk classifiers based on high-dimensional data. Brief Bioinform. 2011;12:203-14.

10. Youden WJ. Index for rating diagnostic tests. Cancer. 1950;3: 32-5.

11. Heagerty PJ, Lumley T, Pepe MS. Time-dependent ROC curves for censored survival data and a diagnostic marker. Biometrics. 2000;56:337-44.

12. Sorbellini M, Kattan MW, Snyder ME, Reuter V, Motzer R, Goetzl M, et al. A postoperative prognostic nomogram predicting recurrence for patients with conventional clear cell renal cell carcinoma. J Urol. 2005;173:48-51.

13. Chi DS, Palayekar MJ, Sonoda Y, Abu-Rustum NR, Awtrey CS, Huh J, et al. Nomogram for survival after primary surgery for bulky stage IIIC ovarian carcinoma. Gynecol Oncol. 2008;108:191-4.

14. Shariat SF, Karakiewicz PI, Suardi N, Kattan MW. Comparison of nomograms with other methods for predicting outcomes in prostate cancer: a critical analysis of the literature. Clin Cancer Res. 2008;14:4400-7.

15. Cohen JG, Tran AQ, Rimel BJ, Cass I, Walsh CS, Karlan BY, et al. Thrombocytosis at secondary cytoreduction for recurrent ovarian cancer predicts suboptimal resection and poor survival. Gynecol Oncol. 2014;132:556-9.

16. Cho H, Hur HW, Kim SW, Kim SH, Kim JH, Kim YT, et al. Pre-treatment neutrophil to lymphocyte ratio is elevated in epithelial ovarian cancer and predicts survival after treatment. Cancer Immunol Immunother. 2009;58:15-23.

17. Davis AN, Afshar-Kharghan V, Sood AK. Platelet effects on ovarian cancer. Semin Oncol. 2014;41:378-84.

18. Mantovani A, Allavena P, Sica A, Balkwill F. Cancer-related inflammation. Nature. 2008;454:436-44.

19. Milne K, Alexander C, Webb JR, Sun W, Dillon K, Kalloger SE, et al. Absolute lymphocyte count is associated with survival in ovarian cancer independent of tumor-infiltrating lymphocytes. J Transl Med. 2012;10:33.

20. Williams KA, Labidi-Galy SI, Terry KL, Vitonis AF, Welch WR, Goodman A, et al. Prognostic significance and predictors of the neutrophil-to-lymphocyte ratio in ovarian cancer. Gynecol Oncol. 2014;132:542-50.

21. Armaiz-Pena GN, Gonzalez-Villasana V, Nagaraja AS, Rodriguez-Aguayo C, Sadaoui NC, Stone RL, et al. Adrenergic regulation of monocyte chemotactic protein 1 leads to enhanced macrophage recruitment and ovarian carcinoma growth. Oncotarget. 2015;6:4266-73.

22. Stone RL, Nick AM, McNeish IA, Balkwill F, Han HD, Bottsford-Miller J, et al. Paraneoplastic thrombocytosis in ovarian cancer. N Engl J Med. 2012;366:610-8.

23. Clark TG, Stewart M, Rye T, Smyth JF, Gourley C. Validation of a new prognostic index for advanced epithelial ovarian cancer: results from its application to a UK-based cohort. J Clin Oncol. 2007;25:5669-70.

24. Barlin JN, Yu C, Hill EK, Zivanovic O, Kolev V, Levine DA, et al. Nomogram for predicting 5-year disease-specific mortality after primary surgery for epithelial ovarian cancer. Gynecol Oncol. 2012;125:25-30.

25. Rutten MJ, Boldingh JH, Schuit E, Trum H, van Driel W, Mol BW, et al. Development and internal validation of a prognostic model for survival after debulking surgery for epithelial ovarian cancer. Gynecol Oncol. 2014;135:13-8.

26. Gerestein CG, Eijkemans MJ, de Jong D, van der Burg ME, Dykgraaf RH, Kooi GS, et al. The prediction of progressionfree and overall survival in women with an advanced stage of epithelial ovarian carcinoma. BJOG. 2009;116:372-80.

27. Lee CK, Simes RJ, Brown C, Gebski V, Pfisterer J, Swart AM, et al. A prognostic nomogram to predict overall survival in patients with platinum-sensitive recurrent ovarian cancer. Ann Oncol. 2013;24:937-43.

28. Lee CK, Simes RJ, Brown C, Lord S, Wagner U, Plante M, et al. Prognostic nomogram to predict progression-free survival in patients with platinum-sensitive recurrent ovarian cancer. Br J Cancer. 2011;105:1144-50.

29. Teramukai S, Ochiai K, Tada H, Fukushima M; Japan Multinational Trial Organization OC01-01. PIEPOC: a new prognostic index for advanced epithelial ovarian cancer: Japan Multinational Trial Organization OC01-01. J Clin Oncol. 2007;25:3302-6.

30. Bodurka DC, Deavers MT, Tian C, Sun CC, Malpica A, Coleman RL, et al. Reclassification of serous ovarian carcinoma by a 2-tier system: a Gynecologic Oncology Group Study. Cancer. 2012;118:3087-94 